\title{
QUALIDADE FÍSICA DE SOLO ARENOSO EM AMBIENTE SEMIÁRIDO SOB SISTEMA DE INTEGRAÇÃO LAVOURA-PECUÁRIA
}

\author{
SOIL PHYSICAL QUALITY OF ARENOSOL IN THE L SEMIARID ENVIRONMENT \\ UNDER INTEGRATED AGRICULTURAL SYSTEMS \&
CALIDAD FÍSICA DEL SUELO ARENOSO EN AMBIENTES SEMIÁRIDOS BAJO SISTEMA DE INTEGRACIÓN CULTIVO-GANADO \&

Recebido em: 24/11/2021 - Aprovado em: 09/12/2021 - Publicado em: 17/12/2021

http://dx.doi.org/10.18011/bioeng2021v15n4p598-616

Pedro Luan Ferreira da Silva' (pedroluanferreira@gmail.com)

Flávio Pereira de Oliveira² (flavio.oliveira@academico.ufpb.br)

Walter Esfrain Pereira² (walterufpb@yahoo.com.br)

Adriana Ferreira Martins ${ }^{2}$ (biol.adriana@gmail.com)

João Henrique Zonta ${ }^{3}$ (joao-henrique.zonta@embrapa.br)

André Julio do Amaral ${ }^{4}$ (andre.amaral@embrapa.br)

Danillo Dutra Tavares ${ }^{5}$ (danilodutrat@hotmail.com)

Alison José da Silva ${ }^{5}$ (alisonjose1997@gmail.com)

\footnotetext{
${ }^{1}$ Universidade Estadual de Maringá. Maringá, Paraná, Brasil.

2 Universidade Federal da Paraíba. Areia, Paraíba, Brasil.

3 Embrapa Algodão. Campina Grande, Paraíba, Brasil.

${ }^{4}$ Embrapa Solos UEP. Recife, Pernambuco, Brasil.

${ }_{5}^{5}$ Universidade Federal Rural de Pernambuco. Recife, Pernambuco, Brasil.
}

\section{RESUMO}

O objetivo deste estudo foi avaliar a qualidade física de um Neossolo Regolítico sob sistema de integração lavoura-pecuária. O delineamento experimental adotado foi o inteiramente casualizado com 11 tratamentos e 5 repetições: T1- U. decumbens; T2- U. decumbens + Milho; T3- U. brizantha + Milho; T4- Milho; T5Amendoim; T6- Tifton + Milho; T7- Milho + Guandu; T8- Tifton; T9- Floresta; T10- Sorgo e T11- Massai. As amostras indeformadas foram coletadas nas camadas de 0-0,1 e 0,1-0,2 m para a determinação de: porosidade do solo, aeração, densidade do solo, condutividade hidráulica, água disponível e granulometria. Os dados foram analisados por meio da ANOVA e PCA. O solo sob T9 apresentou qualidade física superior aos demais tratamentos avaliados, contudo, melhorias físicas substanciais foram observadas no T1, T2 e T11. Conclui-se que os atributos macroporosidade, condutividade hidráulica saturada e porosidade de aeração estão apresentando melhorias expressivas em função da iLP em solo arenoso.

Palavras-chave: Neossolo. Água disponível. Manejo conservacionista. Fração areia. 


\section{INTRODUÇÃO}

Devido à crescente demanda por alimento, os solos arenosos tem sido cada vez mais utilizados na produção agrícola em nível mundial. De acordo com o WRB-FAO, os solos arenosos compreendem os Neossolos quartazrênicos, regolíticos, litólicos e flúvicos e em alguns casos Planossolos, Gleissolos, Cambissolos, Chernossolos, Latossolos Espodossolos e Argissolos (FAO, 2001). Geralmente, esses solos apresentam teor de areia superior a $50 \%$ e argila menor que $20 \%$ até a camada de $0,3 \mathrm{~m}$ de profundidade (HUANG; HARTEMINK, 2020). Globalmente, os solos arenosos ocupam uma área de 4.990.200,000 hectares, representando $31 \%$ da crosta terrestre do planeta terra (HUANG; HARTEMINK, 2020).

No Brasil, os solos arenosos, também conhecidos como solos leves ocupam cerca de $8 \%$ do território e são especialmente expressivos na região do MATOPIBA, nos estados do Maranhão, Tocantins, Piauí e Bahia, onde representam cerca de $20 \%$ da área (DONAGEMMA et al., 2016). Apesar do potencial agrícola, os solos arenosos ainda representam um desafio em função das limitações físicas, químicas e biológicas por eles apresentadas. Segundo Ramalho Filho e Beek (1995), as principais limitações apresentadas pelos solos arenosos são a baixa fertilidade, alta suscetibilidade à erosão $\mathrm{e}$ lixiviação de poluentes, déficit hídrico, além de elevada suscetibilidade à erosão e perda de potencial produtivo quando comparado a solos de textura pesada.

$O$ atual interesse por pesquisas com solos arenosos é reflexo da busca de novas áreas para a produção de fibras e proteínas, no entanto, a produção de gêneros nesse ambiente deve ser acompanhada por práticas agrícolas sustentáveis com base em sistemas de produção conservacionistas. Os sistemas integrados de produção agropecuária, a exemplo da integração lavoura-pecuária (iLP) e lavoura-pecuária-floresta (iLPF), assim como o sistema plantio direto (SPD), são boas alternativas de produção em solos arenosos e, vem sendo amplamente utilizados na recuperação de pastagens e áreas degradadas no Brasil (BRAVIN; OLIVEIRA, 2014; NICODEMO et al., 2018).

Estimativas apontam que a atual área ocupada com sistemas integrados de produção agropecuária no Brasil é cerca de 11,5 milhões de hectares, com estimativas de aumento de mais 5 milhões de hectares até o ano de 2030 (REDE ILPF, 2019; SKORUPA; MANZATO 2019). Os benefícios que os sistemas integrados de produção podem promover aos solos arenosos são múltiplos, a exemplo de melhorias nas taxas de infiltração e 
retenção de água no solo, incremento de carbono orgânico e desenvolvimento do sistema radicular dos vegetais (PEZARICO et al., 2013), aumento de porosidade e agregação (LOSS et al., 2014) e incremento de biomassa microbiana (MUNIZ et al., 2011). No Agreste nordestino, algumas áreas de pastagens estão inseridas em solos degradados devido a acentuado teor de areia e a ausência de adoção de sistemas conservacionistas de produção.

Dessa forma este trabalho testa a hipótese de que a adoção de sistemas integrados de produção em áreas de pastagens degradadas sobre solos arenosos melhora a qualidade física do solo no médio prazo, além de favorecer o acúmulo de carbono e maior disponibilidade de água. Sendo assim, objetivo desse estudo foi avaliar a qualidade física de um Neossolo Regolítico sob sistema de integração lavoura-pecuária no semiárido da Paraíba.

\section{MATERIAL E MÉTODOS}

\subsection{Caracterização da área experimental}

O experimento foi conduzido em área experimental da Empresa Estadual de Pesquisa, Extensão e Regularização Fundiária da Paraíba (EMPAER), em Lagoa Seca, na mesorregião do Agreste (7010'15" S e 3551'13" W). O clima que predomina no município é do tipo As' (quente e úmido) segundo a classificação Köppen-Geiger, com ocorrência de maior precipitação entre os meses de abril e junho (SILVA et al., 2015). A temperatura média anual varia entre 22 e $26^{\circ} \mathrm{C}$, com umidade relativa dor ar de $79 \%$ e precipitação pluviométrica anual de $990 \mathrm{~mm}$ (OLIVEIRA et al., 2009). O relevo do município é caracterizado como fortemente ondulado, recoberto por remanescentes florestais do tipo subcaducifólia. De acordo com o Sistema Brasileiro de Classificação de solos - Sibics, o solo que predomina na área experimental foi caracterizado como Neossolo Regolítico distrófico de textura areia-franca (SANTOS et al., 2018).

No ano de 2015 implantou-se um sistema de plantio direto (SPD) na área experimental de Lagoa Seca, PB, em parcelas experimentais construídas em terraços, devido o relevo suave-ondulado do local. Foram semeadas diferentes espécies de gramíneas a lanço e na entrelinha, logo após a dessecação semeou-se as sementes de milho sobre a palhada. Realizou-se a adubação na área de forma a atender as necessidades nutricionais das 
culturas, tomando-se como base a análise de solo e, aplicou-se $160 \mathrm{~kg} \mathrm{ha}^{-1}$ do adubo monoamônio fosfato. Foram feitas duas adubações de cobertura nos estádios V5 e V8, utilizando 100 e $150 \mathrm{~kg} \mathrm{ha}^{-1}$ de ureia, respectivamente, além de $80 \mathrm{~kg} \mathrm{ha}^{-1}$ de cloreto de potássio em cobertura (ZONTA et al., 2016). Para o controle das plantas daninhas realizouse a aplicação de herbicidas na área, em torno de 20 a 30 dias após a emergência do milho (Zea mays L.), quando a mesma estiver com 4 a 6 folhas verdadeiras e quando as forrageiras estiverem com o mínimo de 3 perfilhos.

\subsection{Delineamento Experimental e Tratamentos}

O delineamento experimental adotado foi o inteiramente casualizado com onze tratamentos e cinco repetições $(11 \times 5)$. Os tratamentos avaliados foram: 1) Urochloa decumbens $\left(\mathrm{T}_{1}\right)$; 2) Urochloa decumbens + Milho $\left(\mathrm{T}_{2}\right)$; 3) Urochloa brizantha + Milho ( $\left.\mathrm{T}_{3}\right)$; 4) Milho Solteiro $\left(T_{4}\right)$; 5) Amendoim $\left(T_{5}\right)$; 6) Capim Tifton + Milho $\left(T_{6}\right)$; 7$)$ Milho + Guandu $\left(T_{7}\right)$; 8) Tifton ( $\left.T_{8}\right)$; 9) Floresta $\left(T_{9}\right)$; 10) Sorgo forrageiro ( $\left.T_{10}\right)$ e 11) Capim Massai ( $\left.T_{11}\right)$.

\subsection{Atributos determinados}

As amostras de solo com estrutura indeformada e deformada foram coletadas no ano de 2018 em duas profundidades amostrais (0-0,1 e 0,1-0,2 m) em todas as parcelas experimentais, quando as culturas estavam em estádio pleno de desenvolvimento, próximo à época de floração, em malha regular amostral com 10 metros de distância entre os pontos. Para a coleta das amostras indeformadas utilizou-se cilindros metálicos tipo Uhland com volume conhecido de $98,17 \mathrm{~cm}^{-3}$, enquanto que para as amostras deformadas, utilizou-se pá reta de corte.

As coletas totalizaram 111 amostras de solo com estrutura preservada, que foram utilizadas para a obtenção dos seguintes atributos: densidade do solo (Ds, $\mathrm{Mg} \mathrm{m}^{-3}$ ), porosidades (Total (PT), macroporosidade (Ma) e microporosidade (Mi); $\mathrm{m}^{3} \mathrm{~m}^{-3}$ ), porosidade de aeração $\left(\mathrm{Pa}-\mathrm{m}^{3} \mathrm{~m}^{-3}\right)$, condutividade hidráulica saturada $\left(\mathrm{K}_{\theta} \mathrm{cm} \mathrm{h}^{-1}\right)$, capacidade de campo $\left(\theta_{c c}, \mathrm{~m}^{3} \mathrm{~m}^{-3}\right)$, ponto de murcha permanente $\left(\theta_{\mathrm{PMP}}, \mathrm{m}^{3} \mathrm{~m}^{-3}\right)$ e água disponível $\left(\theta_{\mathrm{AD}}, \mathrm{m}^{3} \mathrm{~m}^{-3}\right)$. As amostras com estrutura não preservada (66), foram utilizadas na obtenção de: Areia, Silte, Argila, Argila dispersa em água (ADA) e o grau de floculação do solo (GF) em $\mathrm{g} \mathrm{kg}^{-1}$. A densidade do solo (Ds), foi determinada através da relação entre a massa do solo seco/ volume (BLAKE; HARTGE, 1986). 
A porosidade total (PT) foi determinada pelo volume de saturação do solo, conforme descrito no manual de métodos e análise de solos da EMBRAPA (TEIXEIRA et al., 2017). A microporosidade do solo (Mi) foi obtida em mesa de tensão com umidade correspondente ao potencial de -6 kPa. Por diferença (PT-Mi), obteve-se a macroporosidade do solo (Ma). A condutividade hidráulica saturada $\left(\mathrm{K}_{\theta}\right)$, foi determinada com o auxílio do permeâmetro de carga constante, conforme descrito por Teixeira et al. (2017) (Eq. 1)

$\mathrm{K}_{\theta}=\frac{(\mathrm{Q} \times L)}{(A \times H \times T)}$

onde: $K_{\theta}$ é a condutividade hidráulica saturada $\left(\mathrm{cm} \mathrm{h}^{-1}\right), \mathrm{Q}$ - volume percolado coletado em proveta $\left(\mathrm{mL}^{-1}\right), \mathrm{L}$ - altura do bloco de solo em $(\mathrm{cm}), \mathrm{A}$ - área do cilindro em $\left(\mathrm{cm}^{2}\right), \mathrm{H}$ - (altura do bloco de solo + coluna de água $(\mathrm{cm})$ e T - (tempo de coleta do volume de água percolado $\left.h^{-1}\right)$.

A porosidade de aeração $(\mathrm{Pa})$, foi obtida conforme descrito por Reynolds et al. (2007), utilizando a (Eq. 2).

$\mathrm{Pa}=\frac{\theta s}{\theta c c}$

onde: $\theta \mathrm{s}$ - umidade volumétrica da amostra de solo saturada $\left(\mathrm{m}^{3} \mathrm{~m}^{-3}\right) ; \theta c c$ - capacidade de campo $\left(\mathrm{m}^{3} \mathrm{~m}^{-3}\right)$ em -10 kPa. Para a obtenção da $\theta_{\mathrm{cc}}$ e do $\theta_{\mathrm{PMP}}$, as amostras foram saturadas e submetidas aos potenciais matriciais de $\psi-10 \mathrm{kPa}$ em mesa de tensão e, $-1500 \mathrm{em}$ câmara de Richards com placas porosas, respectivamente (KLUTE, 1972). Através da relação $\theta_{\mathrm{CC}}$ - $\theta_{\mathrm{PMP}}$, calculou-se a água disponível no solo $\left(\theta_{\mathrm{Ad}}, \mathrm{m}^{3} \mathrm{~m}^{-3}\right)$.

A granulometria foi determinada através do método do densímetro, utilizando o Hidróxido de Sódio ( $\mathrm{NaOH}-1 \mathrm{~mol} \mathrm{~L}^{-1}$ ) (GEE; BAUDER, 1986), como dispersante químico. Para a argila dispersa em água (ADA), utilizou-se o mesmo procedimento da determinação da argila total, porém, sem o uso do dispersante químico (TEIXEIRA et al., 2017). A partir dos dados da argila total e dispersa, foi possível calcular o grau de floculação do solo (GF). A areia retida na peneira de $0,053 \mathrm{~mm}$, foi fracionada em classes de tamanho utilizando um separador mecânico com peneiras de malha $(1,0 ; 0,50 ; 0,25 ; 0,105$ e $0,053 \mathrm{~mm})$, para a obtenção das areias: muito grossa (AMG), grossa ( $A G)$, média (AM), fina (AF) e muito fina (AMF). 
2.4 Procedimentos estatísticos

Os resultados obtidos na determinação dos atributos físicos foram avaliados por meio de análise de variância e correlação de Pearson (r). Comparações de médias foram efetuadas com aplicação do teste de Tukey a $5 \%$ de probabilidade. Foi utilizado 0 procedimento MIXED com pseudo-repetições SAS/STAT (SAS, 2011) e a análise de componentes principais, utilizando o software $R$ (R DEVELOPMENT CORE TEAM, 2013).

\section{RESULTADOS E DISCUSSÃO}

A classificação textural, a granulometria da fração areia e o grau de floculação (GF) encontram-se apresentados na Tabela 1. Verifica-se que houve diferença significativa para Argila na camada de 0-0,1 m ( $<<0,05)$. Para os demais atributos não houve diferença entre os tratamentos e profundidades avaliadas. 
Tabela 1. Granulometria, argila dispersa em água (ADA) e grau de floculação (GF) em solo sob sistema de integração Lavoura-Pecuária no Agreste paraibano.

\begin{tabular}{|c|c|c|c|c|c|c|c|c|c|c|}
\hline \multirow{2}{*}{ Sistemas } & Areia & Silte & Argila & AMG & $\mathbf{A G}$ & AME & AF & AMF & ADA & GF \\
\hline & \multicolumn{10}{|c|}{ - } \\
\hline & \multicolumn{10}{|c|}{$0-0,1 \mathrm{~m}$} \\
\hline $\mathrm{T} 1$ & $810 a$ & $90 a$ & $100 a b$ & $38 a$ & $205 a$ & 269 a & $219 a$ & $81 \mathrm{a}$ & $0 \mathrm{a}$ & $1000 a$ \\
\hline T2 & 849 a & $89 \mathrm{a}$ & $62 \mathrm{~b}$ & $47 \mathrm{a}$ & $232 \mathrm{a}$ & 278 a & $221 \mathrm{a}$ & $71 \mathrm{a}$ & $40 \mathrm{a}$ & $940 \mathrm{a}$ \\
\hline T3 & 796 a & $91 \mathrm{a}$ & $113 a b$ & $40 \mathrm{a}$ & $192 \mathrm{a}$ & $252 \mathrm{a}$ & $231 \mathrm{a}$ & $81 \mathrm{a}$ & $40 \mathrm{a}$ & $920 \mathrm{a}$ \\
\hline T4 & $830 a$ & $91 \mathrm{a}$ & 79 b & $39 a$ & $212 a$ & $283 a$ & $225 a$ & $69 a$ & $0 \mathrm{a}$ & $1000 a$ \\
\hline T5 & $796 a$ & $102 \mathrm{a}$ & $102 a b$ & $40 \mathrm{a}$ & $202 \mathrm{a}$ & $265 a$ & $220 a$ & $69 a$ & $160 \mathrm{a}$ & $820 \mathrm{a}$ \\
\hline T6 & $816 a$ & $88 \mathrm{a}$ & $112 a b$ & $46 a$ & $223 a$ & $274 a$ & $210 a$ & $63 a$ & $170 \mathrm{a}$ & $860 a$ \\
\hline T7 & $821 \mathrm{a}$ & $90 \mathrm{a}$ & $90 \mathrm{~b}$ & $45 a$ & $213 a$ & $268 \mathrm{a}$ & $225 a$ & $70 \mathrm{a}$ & $80 a$ & $920 \mathrm{a}$ \\
\hline T8 & 793 a & $98 \mathrm{a}$ & $108 a b$ & $34 \mathrm{a}$ & $175 a$ & $256 \mathrm{a}$ & $241 \mathrm{a}$ & $88 \mathrm{a}$ & $80 a$ & $930 \mathrm{a}$ \\
\hline T9 & $817 a$ & $89 a$ & $94 a b$ & $44 a$ & $214 a$ & $267 \mathrm{a}$ & $216 a$ & $75 a$ & $200 \mathrm{a}$ & $810 a$ \\
\hline T10 & $774 \mathrm{a}$ & $77 \mathrm{a}$ & 149 a & $53 a$ & $207 a$ & $243 a$ & $197 \mathrm{a}$ & $74 \mathrm{a}$ & $70 \mathrm{a}$ & $900 \mathrm{a}$ \\
\hline T11 & $784 a$ & $111 a$ & $101 a b$ & $47 a$ & $198 a$ & $248 a$ & $218 a$ & $73 a$ & $40 a$ & $930 a$ \\
\hline \multirow[t]{2}{*}{$\mathrm{CV}(\%)$} & 3,26 & 18,87 & 18,76 & 21,61 & 10,57 & 6,70 & 11,13 & 14,38 & 128,32 & 12,30 \\
\hline & \multicolumn{10}{|c|}{$0,10-0,20 \mathrm{~m}$} \\
\hline $\mathrm{T} 1$ & $819 a$ & $90 \mathrm{a}$ & $92 \mathrm{a}$ & $50 a$ & $214 a$ & $257 a$ & $228 a$ & $70 \mathrm{a}$ & $90 \mathrm{a}$ & $810 \mathrm{a}$ \\
\hline $\mathrm{T} 2$ & $837 \mathrm{a}$ & $97 \mathrm{a}$ & $67 a$ & $43 a$ & $217 \mathrm{a}$ & $259 a$ & $239 a$ & $80 a$ & $210 a$ & $900 \mathrm{a}$ \\
\hline T3 & $787 \mathrm{a}$ & $109 a$ & $105 \mathrm{a}$ & $43 a$ & $206 a$ & $245 a$ & $223 a$ & $70 \mathrm{a}$ & $210 \mathrm{a}$ & $770 \mathrm{a}$ \\
\hline $\mathrm{T} 4$ & $839 a$ & $87 a$ & $73 a$ & $54 \mathrm{a}$ & $247 a$ & $259 a$ & $221 \mathrm{a}$ & $58 \mathrm{a}$ & $170 \mathrm{a}$ & $750 a$ \\
\hline T5 & $821 \mathrm{a}$ & $100 \mathrm{a}$ & $78 \mathrm{a}$ & $41 \mathrm{a}$ & $203 a$ & $265 a$ & $242 \mathrm{a}$ & $69 a$ & $210 \mathrm{a}$ & $710 \mathrm{a}$ \\
\hline T6 & $773 \mathrm{a}$ & $94 a$ & $133 a$ & $57 a$ & $214 a$ & $229 a$ & $221 \mathrm{a}$ & $52 \mathrm{a}$ & $300 \mathrm{a}$ & $740 a$ \\
\hline $\mathrm{T7}$ & $726 \mathrm{a}$ & $117 \mathrm{a}$ & $157 \mathrm{a}$ & $40 \mathrm{a}$ & $192 \mathrm{a}$ & $235 a$ & $205 a$ & $54 a$ & $130 \mathrm{a}$ & $900 \mathrm{a}$ \\
\hline T8 & $778 a$ & $99 \mathrm{a}$ & $123 a$ & $37 \mathrm{a}$ & $176 \mathrm{a}$ & $261 a$ & $210 a$ & $90 \mathrm{a}$ & $340 \mathrm{a}$ & $680 a$ \\
\hline T9 & $775 a$ & $104 \mathrm{a}$ & $121 \mathrm{a}$ & $38 \mathrm{a}$ & $193 a$ & $240 a$ & $225 \mathrm{a}$ & $79 a$ & $300 \mathrm{a}$ & $770 \mathrm{a}$ \\
\hline T10 & $792 \mathrm{a}$ & $102 \mathrm{a}$ & $106 \mathrm{a}$ & $59 a$ & $204 a$ & $243 a$ & $209 a$ & $77 \mathrm{a}$ & $250 \mathrm{a}$ & $810 a$ \\
\hline T11 & $805 a$ & $96 a$ & $99 a$ & $38 \mathrm{a}$ & $205 a$ & $257 a$ & $230 a$ & $75 a$ & $170 \mathrm{a}$ & $840 a$ \\
\hline CV (\%) & 5,84 & 24,85 & 34,46 & 25,13 & 16,06 & 9,57 & 16,84 & 39,65 & 49,56 & 11,99 \\
\hline
\end{tabular}

Urochloa decumbens $\left(T_{1}\right)$; Urochloa decumbens + Milho $\left(T_{2}\right)$; Urochloa brizantha + Milho $\left(T_{3}\right)$; Milho Solteiro $\left(T_{4}\right)$; Amendoim ( $\left.T_{5}\right)$; Capim Tifton + Milho ( $\left.T_{6}\right)$; Milho + Guandu $\left(T_{7}\right)$; Tifton ( $\left.T_{8}\right)$; Floresta $\left(T_{9}\right)$; Sorgo forrageiro $\left(T_{10}\right)$ e Capim Massai ( $\left.T_{11}\right)$. AMG - (Areia muito grossa); AG - (Areia grossa); AME - (Areia média); AF - (Areia fina); AMF - (Areia muito fina). Letras diferentes na coluna diferem pelo teste de Tukey $(p<0,05)$.

Maior concentração de argila foi verificada no tratamento SRG $\left(149 \mathrm{~g} \mathrm{~kg}^{-1}\right)$ e menor no tratamento $B D+M l\left(62 \mathrm{~g} \mathrm{~kg}^{-1}\right)$. Esse resultado não foi influenciado pelo sistema de manejo implantado, pois, a textura é um atributo estável do solo, intrínseco, e só pode ser modificado através de processos naturais como o intemperismo químico, físico e biológico do solo. Além disso, as diferenciações texturais entre SRG e os demais sistemas avaliados podem estar relacionados com maiores ou menores perdas laterais, em superfície, que se processam por arraste mecânico ou dissolução, em função de seu posicionamento do relevo (PARAHYBA et al., 2019).

Contudo, maior concentração de areia no Neossolo principalmente em sua fração grosseira favorece maior aeração do solo, porém, reduz a capacidade de armazenamento 
e disponibilidade de água às plantas, ocasionando déficit hídrico no período de estiagem. Comportamento similar foi verificado por Silva et al. (2018) em Neossolos e Argissolos com teor de areia > 90\% na camada de 0-20 cm. Nesse sentido, o manejo dos solos arenosos deve ser realizado através de práticas conservacionistas de produção. Para Gazzola et al. (2015), a adoção de sistemas integrados de produção agropecuária em solos arenosos promove a manutenção de resíduos vegetais em superfície, melhorando a qualidade física, química e biológica do solo.

O GF foi superior em superfície e reduziu com o aumento de profundidade do solo, demonstrando que em superfície a floculação de argila é mais adequada que em subsuperfície, tornando o solo mais estável (Tabela 1). Esse comportamento deve ser oriundo do aporte de $\mathrm{MO}$ em superfície, elemento esse que atua sobre a floculação da argila em solos. Um dos benefícios do elevado GF é que a probabilidade de ocorrer problemas de obstrução de poros por movimentação de argila é menor (MOTA et al., 2015), reduzindo as chances de ocorrer processo erosivo por ocasião de selamento da camada superficial do solo.

$\mathrm{Na}$ Tabela 2, estão apresentados os valores médios de porosidade total, macroporosidade, microporosidade, porosidade de aeração, densidade do solo, condutividade hidráulica saturada, capacidade de campo e ponto de murcha permanente. Observa-se que houve diferença significativa para MA, PA e $\mathrm{K}_{\theta}$ na camada de 0-0,1 m. Na camada de 0,1-0,2 m não foi observado diferença significativa para $K_{\theta}$, $\theta$ cc e $\theta$ PMP. 
Tabela 2. Valores médios de porosidade total (PT), macroporosidade (Ma), microporosidade (Mi), porosidade de aeração $(\mathrm{Pa})$, densidade do solo $(\mathrm{Ds})$, condutividade hidráulica saturada $\left(\mathrm{K}_{\theta}\right)$ e retenção de água (Capacidade de campo, ponto de murcha permanente e água disponível ( $\left.\theta_{\mathrm{cc}}, \theta_{\mathrm{PMP}}\right)$, em solo sob sistema de integração Lavoura-Pecuária, no Agreste paraibano.

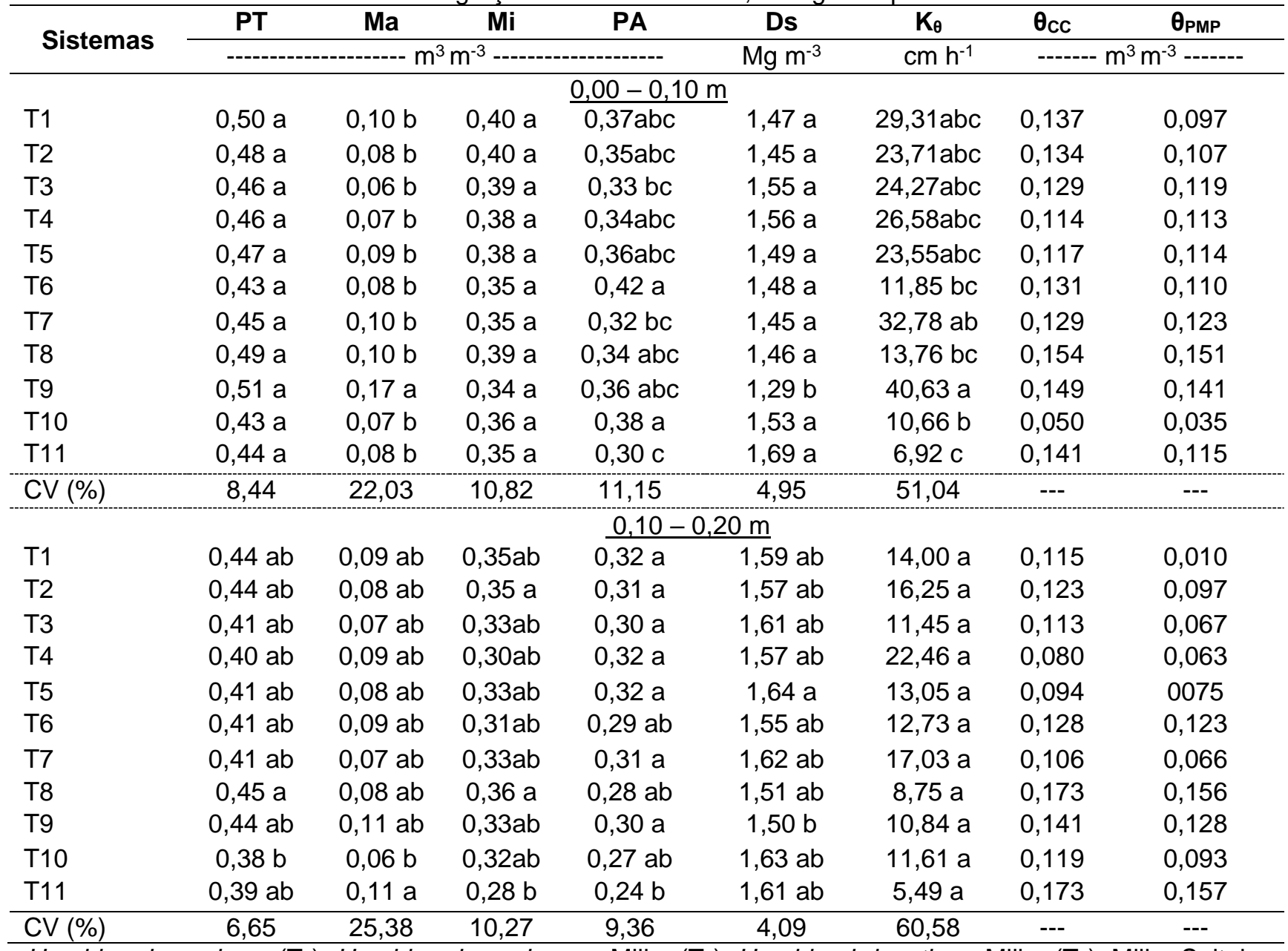

Urochloa decumbens $\left(T_{1}\right)$; Urochloa decumbens + Milho $\left(T_{2}\right)$; Urochloa brizantha + Milho $\left(T_{3}\right)$; Milho Solteiro $\left(T_{4}\right)$; Amendoim ( $\left.T_{5}\right)$; Capim Tifton + Milho $\left(T_{6}\right)$; Milho + Guandu $\left(T_{7}\right)$; Tifton ( $\left.T_{8}\right)$; Floresta $\left(T_{9}\right)$; Sorgo forrageiro $\left(T_{10}\right)$ e Capim Massai $\left(T_{11}\right)$. Trat. - (Tratamentos). Letras diferentes na coluna diferem pelo teste de Tukey $(p<0,05)$.

A macroporosidade foi superior no tratamento FLO $\left(0,17 \mathrm{~m}^{3} \mathrm{~m}^{-3}\right)$ e diferiu dos demais sistemas avaliados (Tabela 2). Essa tendência ocorreu na camada de 0,1-0,2 m e foi superior no tratamento MASSAI $\left(0,11 \mathrm{~m}^{3} \mathrm{~m}^{-3}\right)$. Com relação ao solo sob FLO, valores significativos de Ma podem estar relacionados a melhor qualidade estrutural do solo nesse ambiente. Além do mais, cabe salientar que apenas no tratamento FLO e MASSAI foram observados valores de macroporosidade do solo superiores ao limite crítico de $0,10 \mathrm{~m}^{3} \mathrm{~m}^{-}$ ${ }^{3}$, como destacado por Parahyba et al. (2019), para solos com teor de argila de $0-200 \mathrm{~g} \mathrm{~kg}^{-}$ 1.

Com relação a PT, observa-se melhor média no tratamento $\mathrm{BD}\left(0,45 \mathrm{~m}^{3} \mathrm{~m}^{-3}\right)$ na camada de 0,1-0,2 m, diferindo dos demais tratamentos avaliados (Tabela 2). Nas duas camadas de solo avaliadas os valores médios de PT permaneceram dentro da faixa 
limitante para solos de textura arenosa $\left(0,33-0,60 \mathrm{~m}^{3} \mathrm{~m}^{-3}\right)$, como observado por Bruand et al. (2005). A elevada porosidade total do solo sob os diferentes tratamentos favorece melhorias na infiltração de água e aeração do solo, contudo, por outro lado, reduz à disponibilidade de água as plantas. Corroborando com o trabalho de Fidalski et al. (2013) em solos arenosos da região do Arenito Caiuá. Para Arcoverde et al. (2015), os solos que apresentam $\mathrm{PT}<0,35 \mathrm{~m}^{3} \mathrm{~m}^{-3}$, são classificados como inapropriados para à agricultura.

A microporosidade só diferiu na camada de 0,1-0,2 m, sendo superior nos tratamentos $\mathrm{BD}+\mathrm{Ml}$ e BD (Tabela 2). Esse resultado demonstra que em consórcio ou solteira, o sistema com BD Urochloa decumbens exerceu influência sobre a MI. Os demais tratamentos foram iguais. As gramíneas, por apresentarem sistema radicular agressivo contribuem para a melhoria da qualidade física do solo. Yu et al. (2018), por exemplo, observaram que a densidade de raízes no sistema apresentou correlação positiva e linear com os atributos porosidade do solo e porosidade não-capilar (macroporos).

A retenção de água no solo analisada pela capacidade de campo e ponto e murcha permanente indicou que os solos apresentam baixa disponibilidade de água para as plantas, principalmente em função dos valores de água disponível abaixo de 14\% (0,140 $\mathrm{m}^{3} \mathrm{~m}^{-3}$ ), que é observado por Reynolds et al. (2007), limitante para a maioria das culturas cultivadas em solos arenosos. Contudo, dentre os tratamentos avaliados, o BD demonstrou valores satisfatórios em ambas as camadas avaliadas (Tabela 2), contudo, abaixo do que seria aceito como suportável para o desenvolvimento dos vegetais.

A porosidade de aeração $(\mathrm{Pa})$, diferiu nas duas camadas de solo entre os tratamentos avaliados. Foi superior nos tratamentos CU+MI e SRG na camada de 0-0,1 m e, nos tratamentos MS e CU na camada de 0,1-0,2 m (Tabela 2). São valores médios que expressam a influência das frações de areia na capacidade de aeração do solo. Contudo, como observado por Silva et al. (2018), em solos arenosos sob iLPF, valores de Pa $>0,34$ $\mathrm{m}^{3} \mathrm{~m}^{-3}$ podem indicar que a acentuada aeração desse solo está afetando a capacidade de armazenamento de água no mesmo.

Com relação a Ds (Tabela 2), menor valor foi verificado no tratamento FLO (1,29 Mg $\mathrm{m}^{-3}$ ) na camada de 0-0,1 m. Conforme observado em trabalho de Bruand et al. (2005), esse valor está dentro da faixa limite para solos de textura arenosa que é de 1,10 a 1,78 $\mathrm{Mg} \mathrm{m}^{-}$ 3. Na camada de 0,1-0,2 m, os valores foram superiores aos observados em superfície. Para Huang e Hartemink (2020), a elevação de Ds em solos arenosos até um certo limite 
(não restritivo) pode ser adequado, se comparado a solos de textura média a argilosa, pois pode favorecer a retenção de água e melhoria na disponibilidade de água às plantas.

A condutividade hidráulica saturada foi significativa apenas na camada de $0-0,1 \mathrm{~m}$, sendo superior no tratamento FLO $\left(40,63 \mathrm{~cm} \mathrm{~h}^{-1}\right)$ e menor no tratamento MASSAI $(6,92 \mathrm{~cm}$ $\mathrm{h}^{-1}$ ), ou seja, uma diferença de $82,96 \%$ (Tabela 2). Esse resultado é reflexo da estrutura do solo e da elevada porosidade total encontrada nesses solos em superfície. Como observado por Silva et al. (2018) há uma estreita relação entre a Kө e a textura do solo. Ribeiro et al. (2017) avaliando as propriedades físicas de seis classes de solo de Minas Gerais, observaram que o volume de macroporos do solo é diretamente proporcional aos valores de condutividade hidráulica saturada, assim como, 0 movimento e 0 armazenamento de água. A $\mathrm{K}_{\theta}$ é sensível as mudanças de macroporosidade no solo, havendo dessa forma maior tendência para valores elevados de $\mathrm{K}_{\theta}$ em superfície (MARQUES et al., 2008).

A manutenção de cobertura morta em superfície como ocorre em solos sob sistemas integrados de produção agropecuária, pode favorecer o aumento da taxa de infiltração de água no solo. E esse comportamento foi observado e validado por Cui et al. (2021) em solos arenosos com biocrostas; os autores concluíram que a manutenção da serapilheira em superfície aumentou a taxa de infiltração de água no solo de 215 a $280 \%$. Para eles isso se deve ao fato de que a manutenção de serapilheira melhora a porosidade nas biocrostas em superfície. Dessa forma, a cobertura promovida pelo iLPF além de contribuir para a melhoria da qualidade estrutural do solo, pode, ao longo do tempo, elevar o potencial produtivo de solos arenosos.

Na Tabela 3 está apresentada a análise de componentes principais para o Neossolo Regolítico sob os sistemas integrados de produção agropecuária. Para os atributos físicos do solo foi observado que $67,96 \%$ da variância dos dados foi explicada pelo CP1, enquanto que $19,80 \%$ foi explicada pelo CP2, totalizando (87,76\%). Contudo, para a granulometria, verificou-se que $34,60 \%$ da variância foi explicada pelo CP1 e 19,60\% pelo CP2, totalizando $(54,20 \%)$. 
Qualidade física de solo arenoso em ambiente semiárido sob sistema...

Tabela 3. Análise de componentes principais para atributos físicos de solo e granulometria de um Neossolo Regolítico sob sistema de integração Lavoura-Pecuária, Lagoa Seca, PB.

\begin{tabular}{|c|c|c|c|}
\hline \multirow{2}{*}{$\begin{array}{c}\text { Componentes da } \\
\text { Variância }\end{array}$} & \multicolumn{3}{|c|}{ Componentes principais } \\
\hline & Dim 1 & $\operatorname{Dim} 2$ & $\operatorname{Dim} 3$ \\
\hline Variância & 4,078 & 1,188 & 0,432 \\
\hline Proporção \% & 67,964 & 19,808 & 7,194 \\
\hline Acumulada \% & 67,964 & 87,772 & 99,171 \\
\hline \multicolumn{4}{|c|}{ Autovetores } \\
\hline$\alpha$ & 0,4717 & 0,0789 & $-0,4228$ \\
\hline $\mathrm{Ma}$ & 0,2198 & $-0,8159$ & $-0,0536$ \\
\hline $\mathrm{Mi}$ & 0,4096 & 0,4419 & $-0,4362$ \\
\hline Ds & $-0,4537$ & 0,3162 & 0,1343 \\
\hline $\mathrm{K}_{\theta}$ & 0,4133 & 0,1526 & 0,6228 \\
\hline Aeração & 0,4296 & 0,0965 & 0,4376 \\
\hline \multicolumn{4}{|c|}{ Granulometria } \\
\hline Variância & 3,805 & 2,156 & 1,617 \\
\hline Proporção \% & 34,590 & 19,597 & 14,702 \\
\hline Acumulada \% & 34,590 & 54,187 & 68,889 \\
\hline \multicolumn{4}{|c|}{ Autovetores } \\
\hline Areia & 0,4784 & 0,0397 & 0,2689 \\
\hline Silte & $-0,2991$ & $-0,1404$ & $-0,0335$ \\
\hline Argila & $-0,4319$ & 0,0225 & $-0,2916$ \\
\hline AMG & 0,0421 & 0,5983 & $-0,1401$ \\
\hline$A G$ & 0,3591 & 0,4415 & $-0,0472$ \\
\hline AME & 0,3993 & $-0,2457$ & 0,2326 \\
\hline $\mathrm{AF}$ & 0,2494 & $-0,0923$ & $-0,0778$ \\
\hline AMF & $-0,1252$ & $-0,3769$ & 0,4928 \\
\hline ADA & $-0,2807$ & 0,2965 & 0,5006 \\
\hline GF & 0,2192 & $-0,3552$ & $-0,5156$ \\
\hline
\end{tabular}

a-Porosidade total, $\mathrm{Ma}$ - Macroporosidade, Mi - Microporosidade, Ds - Densidade do solo, $\mathrm{K}_{\theta}$ - Condutividade hidráulica saturada, AMG - Areia muito grossa, AG - Areia grossa, AME - Areia média, AF - Areia fina, AMF - Areia muito fina, ADA - Argila dispersa em Água, GF - Grau de floculação da argila.

Observa-se na Figura 1 que houve correlação negativa entre Macroporosidade e densidade do solo no $\mathrm{CP} 1=-0,4537$, com um peso de dispersão menor em relação aos atributos $\mathrm{PT}, \mathrm{Mi}, \mathrm{K}_{\theta}$ e $\mathrm{Pa}$. Isso se deve ao fato de que a macroporosidade do solo é mais expressiva em solos arenosos em que em solos de textura argilosa. É possível observar que maior variância dos dados ocorreu na camada de 0-0,1 m, com destaque para os tratamentos CU, BRA+MI, MS e AM. Essa variância demonstra que os atributos $\mathrm{PT}, \mathrm{Mi}, \mathrm{K}_{\theta}$ e $\mathrm{Pa}$ foram os que apresentaram maior peso dentro do conjunto de dados para os tratamentos avaliados, ou seja, a implantação desses tratamentos está ocasionando mudanças na qualidade física do solo sob CU, BRA+MI, MS e AM, principalmente em superfície, onde se concentra o maior volume de raízes. 
Figura 1. Autovetores da análise de componentes principais para os atributos físicos e granulometria de um Neossolo Regolítico sob sistema de integração Lavoura-Pecuária, Lagoa Seca, PB.

A)

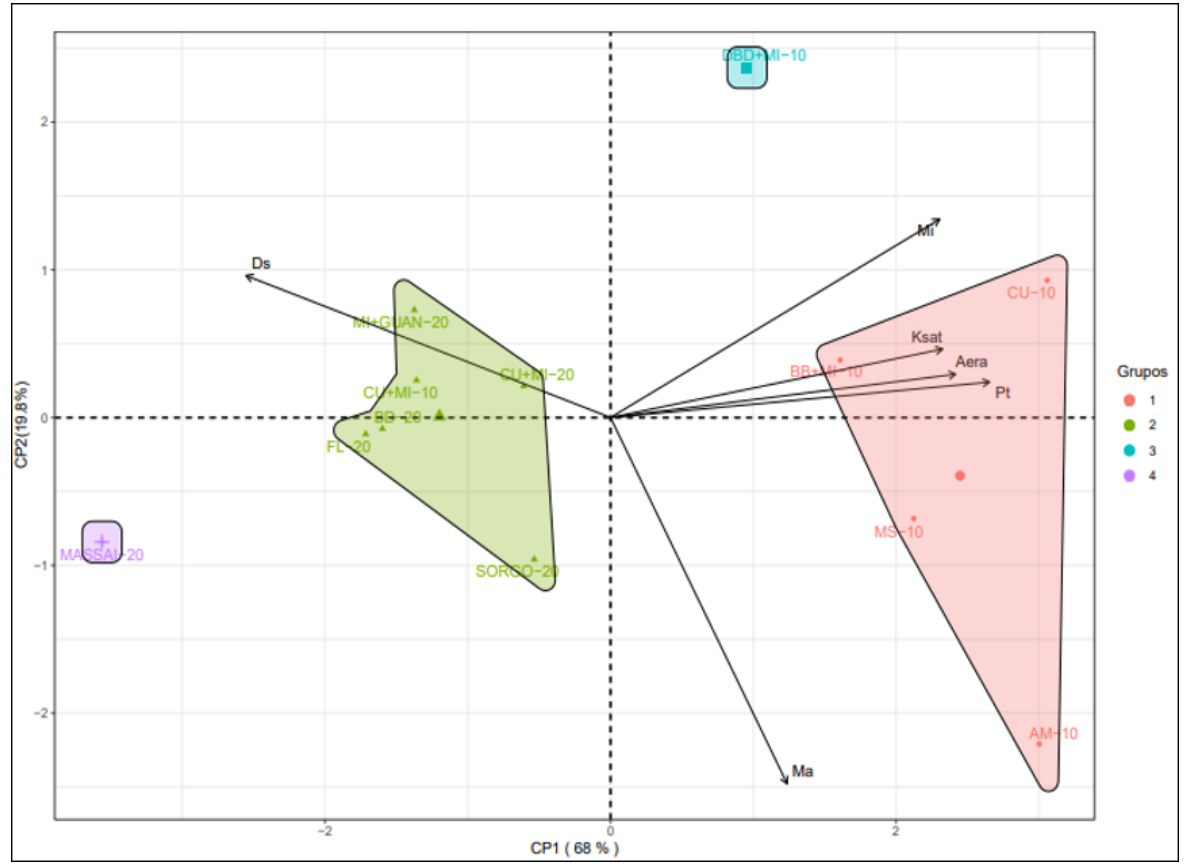

B)

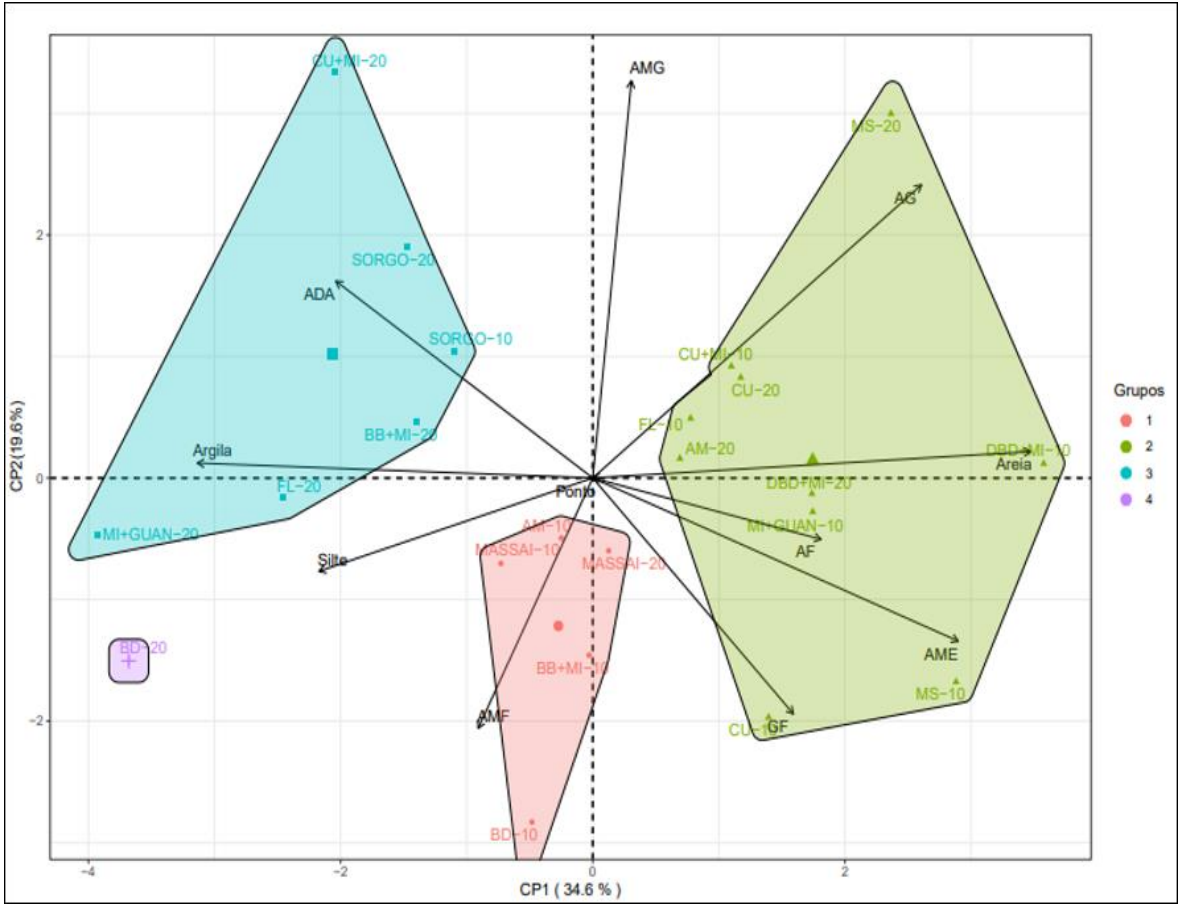

Fonte: Os autores (2021)

Para a Ds, maior peso de pontuação de valores foi observada na camada de 0,1-0,2 $\mathrm{m}$, ocasionalmente para os tratamentos SORGO, FLO, BD, MI+GUAN e, CU+MI em ambas as camadas (Figura 1A). Esse comportamento deve estar relacionado ao aumento no teor de argila em alguns dos tratamentos avaliados com elevação na profundidade do solo. 
Observa-se no PC2 que o escore da Ds aumenta, parecendo mostrar, então que maior parte do peso desse atributo foi retida no PC1.

Atributos como argila e ADA apresentaram relação negativa com o GF e grande parte do peso da Argila dentro do conjunto de dados foi retida no CP2, diferentemente do GF que teve maior parte do seu score ligada ao PC1. Menor score para o PC1 foi observado para a fração mineral areia fina (AF) com 0,2494 (Tabela 3). Esse resultado presume que a AF apresenta menor variabilidade espacial que as demais frações de areia avaliadas, pois os seus scores estão bem próximos a média, diferentemente dos demais. Com base nos tratamentos avaliados, verifica-se que as frações de areia foram mais expressivas na camada de 0-0,1 m, com destaque para os tratamentos MS, CU+MI, CU e AM, assim como o GF com os tratamentos CU e MI. Na camada de 0,1-0,2 m, verifica-se maior influência dos teores de argila e ADA sob os tratamentos SORGO, FLO, CU+MI e MI+GUAN (Figura 1B).

Nas camadas mais profundas do solo a acentuada variabilidade de ADA e argila podem estar relacionadas a mobilização de carbono orgânico por alguns tratamentos com sistema radicular mais profundo e agressivo. Pois, a matéria orgânica do solo em suas frações é responsável por favorecer a melhoria da qualidade estrutural do solo e, bem como influenciar na floculação de argila em solos arenosos. Nesse sentido, afirma-se que alguns dos sistemas integrados de produção agropecuária, nos solos arenosos, estão promovendo algumas alterações físicas principalmente em relação ao tratamento FLO, utilizado aqui como referência de qualidade estrutural do solo.

\section{CONCLUSÕES}

Conclui-se que a adoção de sistemas integrados de produção agropecuária promoveu melhorias físicas a qualidade do solo, com resultados expressivos para macroporosidade, porosidade total, porosidade de aeração e condutividade hidráulica saturada. Apesar do solo sob floresta ter demonstrado qualidade física superior a alguns tratamentos avaliados, conclui-se que melhorias físicas substanciais foram observadas no solo sob os tratamentos capim Massai, Urochloa decumbens e Urochloa decumbes + milho. 


\section{REFERÊNCIAS}

ARCOVERDE, S. N. S.; SALVIANO, A. M.; OLSZEVSKI, N; MELO, S. B.; CUNHA, T. J. F.; GIONGO, V.; PEREIRA, J. S. Qualidade física de solos em uso agrícola na região semiárida do estado da Bahia. Revista Brasileira de Ciência do Solo, Viçosa, Brasil, v. 39, n. 5, p. 1473-1452, 2015. Doi: 10.1590/01000683rbcs20140282

BRUAND, A.; HARTMANN, C.; LESTURGEZ, G. Physical properties of tropical sandy soils: A large range of behaviours. Management of Tropical Sandy Soils for Sustainable Agriculture. A holistic approach for sustainable development of problem soils in the tropics, Thailand: Kohan Kaen, 2005.

BLAKE, G.R.; HARTGE, K.K. Bulk density. In: KLUTE, A. (Ed.). Methods of soil analysis: pat 1: physical and mineralogical methods. $2^{\text {nd }}$ ed. Madison: Soil Science Society of America, 1986, p. 363-382.

BRAVIN, M. P.; OLIVEIRA, T. K. Adubação nitrogenada em milho e capim-xaraés sob plantio direto e preparo convencional em sistema agrossilvipastoril. Pesquisa

Agropecuária Brasileira, Brasília, v. 49, n. 10, p. 762-770, 2014. Doi:10.1590/S0100204X2014001000003

CUI, Z.; HUANG, Z.; LUO, J.; QUIU, K.; LÓPEZ-VICENTE, M.; WU, G.L. Litter cover break soil water repellence of biocrusts, enhancing initial soil water infiltration and content in a semi-arid sandy land. Agricultural Water Management, Amsterdam, Netherlands, 255: 107009, 2021. Doi: 10.1016/j.agwat.2021.107009

DONAGEMMA, G.K.; FREITAS, P.L.; BALIEIRO, F.C.; FONTANA, A.; SPERA, S.T.; LUMBRERAS, J.F.; VIANA, J.H.M.; ARAÚJO FILHO, J.C; SANTOS, F.C.; ALBUQUERQUE, M.R.; MACEDO, M.C.M.; TEIXEIRA, P.C.; AMARAL, A.J.; BORTOLON, E.; BORTOLON, L. Caracterização, potencial agrícola e perspectivas de manejo de solos leves no Brasil. Pesquisa Agropecuária Brasileira, Brasília, Brasil, v. 51, n. 9, p. 10031020, 2016. Doi:10.1590/S0100-204X2016000900001

FIDALSKI, J.; TORMENA, C A.; ALVEZ, S.J.; AULER, P.A.M. Influência das frações de Areia na retenção e disponibilidade de água em solos das formações Caiuá e Paranavaí. Revista Brasileira de Ciência do Solo, Brasília, Brasil, v. 37, n. 3, p. 613-621, 2013. Doi:10.1590/S0100-06832013000300007

FOOD AND AGRICULTURE ORGANIZATION. Lectures notes on the major soils of the world. Rome: FAO, 2001.

GAZOLLA, P. R.; GUARESCHI, R. F.; PERIN, A.; PEREIRA, M. G.; ROSSI, C. Q. Frações de matéria orgânica do solo sob pastagem, sistema plantio direto e integração lavourapecuária. Semina: Ciências Agrárias, Londrina, Brasil, v. 36, n. 2, p. 693-704, 2015. DOI: 10.5433/1679-0359.2015v36n2p693

GEE, G.W.; J.W. BAUDER. Particle Size Analysis. In: Methods of Soil Analysis, Part A. Klute (ed.). $2^{\text {nd }}$ Ed., v. 9, Madison, United States: America Society of Agronomy, 1986. p.383-411. 
HUANG, J.; HARTEMINK, A.E. Soil and environmental issues in sandy soils. EarthScience Reviews, Amsterdam, Netherlands, v. 208, n. 1, p. 1-22, 2020.

https://doi.org/10.1016/j.earscirev.2020.103295

KLUTE, A. Laboratory measurement of hydraulic conductivity of saturated soil. In: BLAKE, C. A. (Ed.). Methods of soil analysis part I. Madison, United States: American Society of Agronomy. 1965p. 210-221. https://doi.org/10.2134/agronmonogr9.1.c13

LOSS, A.; RIBEIRO, E. C.; PEREIRA, M. G.; COSTA, E. M. Atributos físicos e químicos do solo em sistemas de consórcio e sucessão de lavoura, pastagem e silvipastoril em Santa Teresa, ES. Bioscience Journal, Uberlândia, v. 30, n. 5, p. 1347-1357, 2014.

MARQUES, J.D.O.; TEIXEIRA, W.G.; REIS, A.M.; CRUZ JÚNIOR, O.F.; MARTINS, G.C. Avaliação da condutividade do solo saturada utilizando dois métodos de laboratório numa topossequência com diferentes coberturas vegetais no baixo Amazonas. Acta Amazonica, Manaus, Brasil, v. 32, n. 2, p. 193-206, 2008. Doi:10.1590/S004459672008000200002

MOTA, J. C. A.; ALENCAR, T. C.; ASSIS JÚNIOR, R. N. Alterações físicas de um Cambissolo cultivado com bananeira irrigada na capada do Apodi. Revista Brasileira de Ciência do Solo, Viçosa, Brasil, v. 39, p.1015-1024, 2015.

Doi:10.1590/01000683rbcs20140018

MUNIZ, L. C.; MADARI, B. E.; TROVO, J. B. F.; CANTANHÊDE, I. S. L.; MACHADO, P. L. O. A.; COBUCCI, T.; FRANÇA, A. F. S. Soil biological attributes in pastures of different ages in a crop-livestock integrated system. Pesquisa Agropecuária Brasileira, Brasília, v. 46, n. 10, p. 1262-1268, 2011. Doi:0.1590/S0100-204X2011001000021

NICODEMO, M. L. F.; BORGES, W.L.B.; SOUZA, I.M.D. Atributos físicos do solo em quatro sistemas de uso da terra em São Carlos, SP. Revista Brasileira de Ciências Agrárias, Recife, Brasil, v. 13, n. 2, p. 1-7, 2018.

https://doi.org/10.5039/agraria.v13i2a5524

OLIVEIRA, E. M.; SANTOS, M. J.; SOUTO, J. S. Fauna do solo em três ambientes no município de Lagoa Seca - PB. Revista Agropecuária Técnica, Areia, Brasil, v. 30, n. 1, p. 31-36, 2009.

PARAHYBA, R. B. V.; ARAÚJO, M. S. B.; ALMEIDA, B. G.; ROLIM NETO, F. C.; SAMPAIO, E. V. S. B.; CALDAS, A. M. Water retention capacity in Arenososls and Ferralsols in a Semiarid area in the state of Bahia, Brazil. Anais da Academia Brasileira de Ciências, v. 91, n. 4, p. 1-20, 2019. https://doi.org/10.1590/0001-3765201920181031

PEZARICO, C. R.; VITORINO, A. C. T.; MERCANTE, F. M.; DANIEL, O. Indicadores de qualidade do solo em sistemas agroflorestais. Revista de Ciências Agrárias, Belém, Brasil, v. 56, n. 1, p. 40-47, 2013.

R DEVELOPMENT CORE TEAM. R: A language and environment for statistical computing. Vienna: R Foundation for Statistical Computing, 2013. 
RAMALHO FILHO, A.; BEEK, K. J. Sistema de avaliação da aptidão agrícola das terras. 3.ed. Rio de Janeiro: EMBRAPA-CNPS, 1995. 65p.

REDE ILPF. Integração lavoura-pecuária-floresta em números. 2019. Disponível em: https://www.redeilpf.org.br/index.php/rede-ilpf/ilpf-em-numeros. Acesso em: 09 dez. 2021.

REYNOLDS, W.D.; DRURY, C.F.; YANG, X.M.; FOX, C.A.; TAN, C.S.; ZHANG, T. Q. Land management effects on the near-surface physical quality of a clay loam. Soil and Tillage Research, Amterdam, Netherland, v. 96, n. 1-2, p. 316-330, 2007. Doi: 10.1016/j.still.2007.07.003

RIBEIRO, K. D.; MENEZES, S. M.; MESQUITA, M. G. B. F.; SAMPAIO, F. M. T. Propriedades físicas do solo, influenciadas pela distribuição de poros, de seis classes de solos da região de Lavras-MG. Ciência e Agrotecnologia, Lavras, Brasil, v .31, n. 4, p. 1167-1175, 2007. Doi:10.1590/S1413-70542007000400033

SANTOS, H.G.; JACOMINE, P.K.T.; ANJOS, L.H.C.; OLIVEIRA, V.Á.; LUMBRERAS, J.F.; COELHO, M.R.; ALMEIDA, J.A.; ARAÚJO FILHO, J.C.; OLIVEIRA, J.B.; CUNHA, T.J.F. Sistema Brasileiro de Classificação de Solos. Brasília: Embrapa, 2018. 356p.

SAS INSTITUTE. Software SAS STAT versão 9.2. Disponível em:

https://www.sas.com/pt br/home.html

SILVA, G. F.; SANTOS, D.; SILVA, A. P.; SOUZA, J. M. Indicadores de qualidade do solo sob diferentes sistemas de uso na mesorregião do Agreste paraibano. Revista Caatinga, Mossoró, Brasil, v. 28, n. 3, p. 23-35, 2015. Doi:10.1590/1983-21252015v28n303rc

SILVA, P.L.F.; OLIVEIRA, F.P.; BORBA, J.O.M.; TAVARES, D.D.; AMARAL, A.J.; MARTINS, A.F. Solos arenosos para sistemas de integração lavoura-pecuária-floresta em Arez, Rio Grande do Norte. Revista Verde de Agroecologia e Desenvolvimento Sustentável, Pombal, Brasil, v. 13, n. 5, p. 581-589, 2018. Doi:10.18378/rvads.v13i5.6246

SKOPURA, L.A.; MANZATO, C.V. Sistemas de integração Lavoura-Pecuária-Floresta no Brasil. Brasília: Embrapa, 2019. 474p.

TEIXEIRA, P.C.; DONAGEMMA, G.K.; FONTANA. A.; TEIXEIRA, W.G. Manual de métodos e análise de solos. Brasília: Embrapa, 2017. 573p.

YU, B.; XIE, C.; CAI, S.; CHEN, Y.; LV, Y.; MO, Z.; LIU, T.; YANG, Z. Effects of tree roots density on soil porosity and non-capillary porosity using a tree radar unit in Shanghai, China. Suatainability, Berna, Switzerland. 16: 4640, 2018. Doi:10.3390/su10124640

ZONTA, J.H.; SOFIATI, V.; SILVA, O.R.R.F.; RAMOS, C.N.; BARBOSA, H.F.; CORDEIRO JÚNIOR, A.F.; LIRA, A.J.S. Sistema integração Lavoura-Pecuária para a Região Agreste do Nordeste. Campina Grande: Embrapa Algodão, 2016. 26p. 


\begin{abstract}
The objective of this study was to evaluate the physical quality of a Regosol under an integrated crop-livestock system. The experimental design adopted was completely randomized with 11 treatments and 5 replications: T1- U. decumbens; T2- U. decumbens + Maize; T3- U. brizantha + Maize; T4- Corn; T5- Peanut; T6- Tifton + Corn; T7- Corn + Guandu; T8-Tifton; T9- Forest; T10- Sorghum and T11- Massai. Undisturbed samples were collected in the 0-0.1 and 0.1-0.2 m layers to determine: soil porosity, aeration, soil density, hydraulic conductivity, available water and particle size. Data were analyzed using ANOVA and PCA. The soil under T9 presented physical quality superior to the other evaluated treatments, however, substantial physical improvements were observed in T1, T2 and T11. It is concluded that the macroporosity, saturated hydraulic conductivity and aeration porosity attributes are showing significant improvements as a function of iLP in sandy soil.
\end{abstract}

Key-words: Regosol. Water availability. Conservationist management. Sandy fraction.

\title{
RESUMEN
}

El objetivo de este estudio fue evaluar la calidad física de un Regosol bajo un sistema integrado cultivo-ganadería. El diseño experimental adoptado fue completamente al azar con 11 tratamientos y 5 repeticiones: T1- U. decumbens; T2- U. decumbens + Maíz; T3- $U$. brizantha + maíz; T4- Maíz; T5- Maní; T6- Tifton + Maíz; T7- Maíz + Guandu; T8-Tifton; T9Bosque; T10- Sorgo y T11- Massai. Se recolectaron muestras inalteradas en las capas de 0-0.1 y 0.1-0.2 m para determinar: porosidad del suelo, aireación, densidad del suelo, conductividad hidráulica saturada, agua disponible y tamaño de partícula. Los datos se analizaron mediante ANOVA y PCA. El suelo bajo T9 presentó calidad física superior a los otros tratamientos evaluados, sin embargo, se observaron mejoras físicas sustanciales en T1, T2 y T11. Se concluye que los atributos de macroporosidad, conductividad hidráulica saturada y porosidad de aireación están mostrando mejoras significativas en función de iLP en arenosol.

Palabras-Clave: Regosol. Agua disponible. Manejo conservacionista. Fracción Arena. 


\section{LICENÇA DE USO}

Este é um artigo publicado em acesso aberto (Open Access) sob a licença Creative Commons Atribuição 4.0 Internacional (CC BY 4.0), que permite uso, distribuição e reprodução em qualquer meio, desde que o trabalho original seja corretamente citado. Mais informações em: http://creativecommons.org/licenses/by/4.0

\section{CONFLITO DE INTERESSES}

Os autores declaram que não há conflito de interesses neste trabalho.

\section{CONTRIBUIÇÕES AUTORAIS}

Autor 1: Idealização, montagem de experimento, coleta de dados, escrita científica.

Autor 2: Idealização, escrita científica, correção das versões, orientação acadêmica.

Autor 3: Análise e interpretação de dados.

Autor 4: Interpretação de dados e escrita científica.

Autor 5 e 6: Implantação, manutenção da área experimental e viabilização de realização de coletas de dados.

Autor 7 e 8: Coleta de dados, interpretação e escrita científica.

\section{FINANCIAMENTO}

O presente trabalho não contou com apoio financeiro, bolsa de iniciação científica (PIBIC) ao primeiro autor através do CNPq.

\section{COMO REFERENCIAR}

SILVA, Pedro Luan Ferreira da et al. Qualidade física de solo arenoso em ambiente semiárido sob sistema de integração lavoura-pecuária. Revista Brasileira de Engenharia de Biossistemas (Tupã), v. 15, n. 4, p. 598-616, 2021. DOI:

http://dx.doi.org/10.18011/bioeng2021v15n4p598-616.

\section{RESPONSABILIBADE EDITORIAL}

Prof. Dr. Fernando Ferrari Putti ${ }^{1}$, Prof. Dr. Paulo Sérgio Barbosa dos Santos ${ }^{1}$, Prof. Dr. Eduardo Festozo Vicente ${ }^{1}$ e Prof. Dr. Diogo de Lucca Sartori ${ }^{1}$

1 Universidade Estadual Paulista "Júlio de Mesquita Filho", FCE - Faculdade de Ciências e Engenharia, Tupã, SP, Brasil. 